\title{
Downscale cascades in tracer transport test cases: an intercomparison of the dynamical cores in the Community Atmosphere Model CAM5
}

\author{
J. Kent ${ }^{1}$, C. Jablonowski ${ }^{1}$, J. P. Whitehead ${ }^{1,2}$, and R. B. Rood $^{1}$ \\ ${ }^{1}$ Department of Atmospheric, Oceanic and Space Science, University of Michigan, 2455 Hayward St., Ann Arbor, \\ Michigan 48109-2143, USA \\ ${ }^{2}$ Center for Nonlinear Studies, MS B258, Los Alamos National Laboratory, Los Alamos, New Mexico 87545, USA \\ Correspondence to: J. Kent (jdkent@umich.edu)
}

Received: 20 June 2012 - Published in Geosci. Model Dev. Discuss.: 16 July 2012

Revised: 2 November 2012 - Accepted: 19 November 2012 - Published: 6 December 2012

\begin{abstract}
The accurate modeling of cascades to unresolved scales is an important part of the tracer transport component of dynamical cores of weather and climate models. This paper aims to investigate the ability of the advection schemes in the National Center for Atmospheric Research's Community Atmosphere Model version 5 (CAM5) to model this cascade. In order to quantify the effects of the different advection schemes in CAM5, four two-dimensional tracer transport test cases are presented. Three of the tests stretch the tracer below the scale of coarse resolution grids to ensure the downscale cascade of tracer variance. These results are compared with a high resolution reference solution, which is simulated on a resolution fine enough to resolve the tracer during the test. The fourth test has two separate flow cells, and is designed so that any tracer in the western hemisphere should not pass into the eastern hemisphere. This is to test whether the diffusion in transport schemes, often in the form of explicit hyper-diffusion terms or implicit through monotonic limiters, contains unphysical mixing.

An intercomparison of three of the dynamical cores of the National Center for Atmospheric Research's Community Atmosphere Model version 5 is performed. The results show that the finite-volume (CAM-FV) and spectral element (CAM-SE) dynamical cores model the downscale cascade of tracer variance better than the semi-Lagrangian transport scheme of the Eulerian spectral transform core (CAM-EUL). Each scheme tested produces unphysical mass in the eastern hemisphere of the separate cells test.
\end{abstract}

\section{Introduction}

The role of diffusion in dynamical cores of general circulation models (GCMs) is very complex, as it is often used for both physical reasons and numerical reasons (Jablonowski and Williamson, 2011). One area of interest is how dynamical cores represent the effects of subgrid scales. Dynamical cores generally use a fixed grid of finite grid spacing, although there are many different types of grids that can be applied to spherical geometry (Williamson, 2007; Staniforth and Thuburn, 2012). Any scales smaller than the grid spacing cannot be represented explicitly in the dynamical core. Due to the non-linearity of the governing equations small scales can be generated below the grid scale, and these scales interact with the resolved scales. In this paper we investigate the cascade to subgrid scales in the tracer transport component of dynamical cores, focusing on the dynamical cores of the National Center for Atmospheric Research's (NCAR) Community Atmosphere Model version 5 (CAM5) (Neale et al., 2010).

The transport of tracers is an important process in the atmosphere, and needs to be modelled accurately in the dynamical cores of general circulation models. Tracer transport is closely linked to physical parameterizations and chemistry packages. Errors in chemistry models (Prather et al., 2008), or even cloud microphysical parameterizations (Ovtchinnikov and Easter, 2009) may be due to errors in the tracer transport scheme.

Tracer advection algorithms are usually tested on simple test cases, such as constant velocities in one-dimension 
(e.g. Mahlman and Sinclair, 1977; Rood, 1987; Zerroukat et al., 2005; Colella and Sekora, 2008), or solid-body rotation in two (Williamson et al., 1992) or three dimensions (Jablonowski et al., 2008). More complex, deformational flow test cases have recently been developed (Nair and Machenhauer, 2002; Nair and Jablonowski, 2008; Nair and Lauritzen, 2010; Lauritzen et al., 2012) to provide a more challenging test on the sphere. Each of these tests either returns the tracer to its starting position or has an analytical solution, which gives an exact solution that can be used to calculate error norms and convergence rates. To ensure that the final solution is equal to the initial or analytical solution, the tracer must be resolved and there must be no cascade to unresolved scales. These tests are valuable when testing tracer transport schemes, however, the cascade to unresolved scales must also be considered. As the atmosphere is highly non-linear it is possible for tracers to be stretched below the grid scale. Tracer variance, see Sect. 2.2, is conserved in the continuous equations, but cascades downscale to unresolved scales in the discrete case (Thuburn, 2008). The tracer advection scheme must include some sort of "subgrid model" to represent the effects of the unresolved scales and prevent the build up of tracer variance at the grid scale. Usually some sort of diffusion (either explicit or implicit) is deployed to damp the tracer features that are being stretched below the grid scale. This paper will develop prescribed velocity test cases to investigate the tracer cascade in dynamical cores, and to assess how well the diffusion in these dynamical cores models the effects of the subgrid scales.

Many tracer transport algorithms in dynamical cores use constraints to ensure positivity, or filling algorithms to ensure that tracer densities do not become negative. Negative tracer densities are not physical, and can lead to problems in GCM physics parameterizations. Flux and slope limiters are often used with finite-volume methods to try to achieve monotonicity. The inherent diffusion from these limiters and constraints is often used as an implicit "subgrid model" in tracer transport schemes. For example, in the NCAR's Community Atmosphere Model version 5 (CAM5) finite-volume dynamical core (CAM-FV), it is the implicit diffusion due to monotonic limiters that dissipates small scale tracer variance (Lin, 2004; Lin and Rood, 1996). A similar method is also applied in the ECHAM5 model (Roeckner et al., 2003) of the Max Planck Institute for Meteorology. In NCAR's spectral element dynamical core (CAM-SE), explicit hyper-diffusion, positivitypreserving limiters, and monotonic limiters are available (Taylor et al., 2009), and the CAM-SE default configuration employs both a fourth-order hyper-diffusion and a positivedefinite constraint. The UK Met Office model uses implicit diffusion from a semi-Lagrangian scheme with monotonic limiters (Davis et al., 2005; Zerroukat et al., 2002), although explicit hyper-diffusion can also be applied. The Nonhydrostatic ICosahedral Atmospheric Model (NICAM) (Satoh et al., 2008) previously used a second-order centered finite difference method; explicit hyper-viscosity was applied to prevent grid scale noise and a negative fixer was employed to prevent negative tracer densities (Niwa et al., 2011). This is currently being replaced with an up-wind biased flux limiter scheme (Niwa et al., 2011; Miura, 2007; Thuburn, 1996); as with CAM-FV, the inherent diffusion from the flux limiters is used to model the effects of unresolved scales and the downscale cascade of tracer variance.

In this paper, we discuss filtering the governing equations to derive equations for resolved scales and subgrid scales (similar to a large eddy simulation approach Mason, 1994). We develop test cases to investigate how accurately dynamical cores model the cascade to small scales in tracer transport, and whether the diffusion in these dynamical cores is physical. The test cases use prescribed velocities, and although the tests are not "dynamic" they are easily applied to dynamical cores. We perform an intercomparison of the dynamical cores in CAM, to assess their tracer transport diffusion properties, using these test cases. The governing equations and the numerical schemes used with the CAM dynamical cores are described in Sect. 2. Our methodology is explained in Sect. 3, and the tracer test cases are described in Sect. 4. Section 5 shows the results when using the dynamical cores of CAM, and conclusions are drawn in Sect. 6. We focus our attention on two-dimensional non-divergent test cases that generate subgrid scales. Extensions of this work will be the creation of divergent flow test cases, and a set of complex three-dimensional tracer transport tests.

\section{Governing equations and numerical schemes}

\subsection{The continuous equations}

In two-dimensions, the continuity and tracer conservation equation are given as

$$
\begin{gathered}
\frac{\partial \rho}{\partial t}+\nabla \cdot(\rho \boldsymbol{v})=0, \\
\frac{\partial(\rho q)}{\partial t}+\nabla \cdot(\rho \boldsymbol{v} q)=0,
\end{gathered}
$$

where $t$ is time, $\rho$ is the fluid density, $q$ is the tracer mixing ratio and $v$ is the horizontal velocity vector. This gives the advection equation

$$
\frac{\partial q}{\partial t}+\boldsymbol{v} \cdot \nabla q=0
$$

If the fluid is incompressible, and therefore divergence free, $\nabla \cdot v=0$, then Eq. (3) can be written in flux form

$$
\frac{\partial q}{\partial t}+\nabla \cdot(v q)=0
$$

The tests in this paper make use of incompressible flow with prescribed velocities, and a constant density $\rho$, implying that Eqs. (3) and (4) are equivalent. 


\subsection{The discrete equations}

Equations (1)-(4) are the continuous equations, capturing all possible scales. The numerical models used for the atmosphere are not able to capture all of these scales as they are discrete. The scales that can be represented on the model grid are called "resolved" and the scales that cannot be represented on the grid are called "unresolved" or "subgrid". To separate the governing equations into their resolved and unresolved parts we follow the large eddy simulation technique of filtering the equations (Mason, 1994; Grinstein et al., 2007). A filter separates the variables into their resolved and unresolved parts

$q=\bar{q}+q^{\prime}$,

where the bar signifies the spatially filtered part (in this paper we use an area average), and the prime the unresolved parts. The filter can be used to rewrite the continuous equation in terms of filtered variables and a subgrid term

$\frac{\partial \bar{q}}{\partial t}+\overline{\boldsymbol{v}} \cdot \nabla \bar{q}=\overline{\boldsymbol{v}} \cdot \nabla \bar{q}-\overline{\boldsymbol{v} \cdot \nabla q}$.

Note that although we can separate the variables using Eq. (5), we do not make use of the prime variables in this filtered equation. The left hand side of Eq. (6) is the advection equation composed of resolved scale variables, i.e. the variables that are available on our computational grid. The subgrid term is the right hand side of Eq. (6). In atmospheric modeling the tracer transport scheme solves the left hand side of the equation (resolved scales), while some sort of diffusion (either explicit or implicit in the numerical scheme) is used to handle the subgrid scales. In many dynamical cores this diffusion is applied in an ad hoc way, with no physical motivation (Jablonowski and Williamson, 2011).

Tracer variance is defined as

$Z=\int_{A} \rho(q-\langle q\rangle)^{2} d A$,

where $\langle q\rangle$ is the global mean of $q$, and $d A$ is an area element of the domain. In the continuous equation tracer variance is a conserved quantity. However, in the discrete case tracer variance is defined as

$\bar{Z}=\int_{A} \bar{\rho}(\bar{q}-\langle\bar{q}\rangle)^{2} d A$,

and is not conserved (due to the right hand side of Eq. (6)). Tracer variance cascades downscale from large to small scales, and in the discrete case will cascade from resolved to unresolved scales (Thuburn, 2008). Therefore, to accurately model the subgrid terms of the tracer equation, tracer variance must be dissipated, to avoid the accumulation of tracer variance at the grid scale.

\subsection{Horizontal tracer advection schemes in CAM}

We perform an intercomparison of the horizontal tracer transport algorithms in NCAR's Community Atmosphere Model. This will demonstrate the ability of the advection algorithms in CAM to model the downscale cascade of tracer variance. We use the finite-volume dynamical core (CAM-FV) (Lin, 2004), the spectral transform Eulerian dynamical core with semi-Lagrangian tracer transport (CAM-EUL) and the spectral element dynamical core (CAM-SE) (Dennis et al., 2012). This represents the default dynamical core of CAM versions 4 and 5 (CAM-FV), the previous default of CAM version 3 (CAM-EUL), and the default dynamical core from winter 2012 (CAM-SE), of NCAR's Community Earth System Model (CESM1) (Neale et al., 2010).

The horizontal tracer transport scheme in CAM-FV is based around solving the tracer conservation Eq. (2) using the Lin-Rood scheme (Lin and Rood, 1996) on a latitudelongitude grid. The Lin-Rood scheme makes use of multiple one-dimensional operators to solve the two-dimensional problem (see Lin and Rood, 1996, 1997). These onedimensional operators are the difference of numerical fluxes (representing the second term in Eq. 4), and there are many different schemes that can be used to calculate the fluxes (Kent et al., 2012). The default option in CAM-FV is the Piecewise Parabolic Method (PPM) (Colella and Woodward, 1984) with the "default" limiter (given in Appendix B of Lin, 2004). To highlight the characteristics of certain schemes, we will also use the following methods to calculate the numerical fluxes:

- A first-order upwind scheme.

- The van Leer scheme with the monotonized central (MC) limiter (van Leer, 1977).

- The Lax-Wendroff scheme (Lax and Wendroff, 1960).

The first-order and van Leer schemes are both diffusive, and are options in the latest CAM5 release. They are used in these tests to consider whether diffusion can accurately capture the cascade to subgrid scales. The Lax-Wendroff scheme is dispersive and is used to illustrate the effects of a non-dissipative (to leading order) scheme (note that the LaxWendroff scheme is not available in the standard CAM5). CAM-FV makes use of a filling algorithm (Neale et al., 2010), to ensure that tracer mixing ratios are positive definite. This is because the Lin-Rood scheme does not guarantee monotonicity; the Lin-Rood scheme does not properly limit the cross-terms, and this can lead to small over and undershoots. We make use of the filling algorithm for each of the schemes used with CAM-FV, except the Lax-Wendroff scheme. For CAM-FV on the $2^{\circ} \times 2^{\circ}$ resolution grid, which corresponds to a grid spacing of about $220 \mathrm{~km}$ at the equator, we use a tracer time step of $90 \mathrm{~s}$.

The Eulerian spectral transform dynamical core, CAMEUL, employs a two-dimensional semi-Lagrangian scheme 
for horizontal tracer transport on a Gaussian grid. The scheme uses limiters to ensure monotonicity (Williamson and Rasch, 1989, 1994), and does not apply any explicit diffusion mechanisms (note that there is implicit diffusion from the monotonic limiters). CAM-EUL solves the advective form of the transport Eq. (3). The CAM-EUL grid uses a spectral triangular truncation of T85 with a $128 \times 256$ Gaussian transform grid (giving an approximate equatorial grid spacing of $156 \mathrm{~km}$ ), and we use a tracer time step of $150 \mathrm{~s}$.

CAM-SE makes use of the cubed sphere grid (Sadourny, 1972; Rancic et al., 1996), and is built on the spectral element approach (Taylor et al., 1997, 2008; Taylor, 2011). For tracer advection both a sign-preserving limiter (positivedefinite) and explicit fourth-order hyper-diffusion are applied (Taylor et al., 2009). The default coefficient for hyperdiffusion is used (i.e. $6 \times 10^{15} \mathrm{~m}^{4} \mathrm{~s}^{-1}$ on the nel6np4 grid, which corresponds to a grid spacing of about $200 \mathrm{~km}$ ). A time step of $90 \mathrm{~s}$ is used for the ne16np4 grid. To interpolate CAM-SE from the cubed sphere to the latitude-longitude grid, for direct comparison with the other two dynamical cores, we use the Geometrically Exact Conservative Remapping (GECoRe) (Ullrich et al., 2009) algorithm.

\section{Methodology}

To determine how accurately a numerical scheme has modelled the subgrid term, a comparison with a "true" solution needs to be made. Therefore, the tests will be simulated on a coarse grid to demonstrate how the advection scheme models the subgrid scales, and also on a grid with adequate resolution to capture all of the scales providing a reference solution. The reference solution can be averaged/filtered to the coarse grid to evaluate the advective scheme's subgrid model. To determine the accuracy of the reference solution, the numerical scheme and the grid resolution should be tested on an already established resolved scale two-dimensional test case. The deformation test case number 4 given in Nair and Lauritzen (2010) for two-dimensional flow is a suitable example. The resolution that will be used for calculating the reference solution in our subgrid scale tests must be the same resolution that is used when calculating the accuracy of the reference solution on the deformation test of Nair and Lauritzen (2010). The deformation test of Nair and Lauritzen (2010) is designed so that the whole tracer is resolved during the simulation, and the flow is reversed so that error norms can be calculated using the initial conditions. The normalized error norms can be used to give bounds on the accuracy of the reference solution at the tested resolution, and hence error bounds on the accuracy of the scheme on these subgrid scale tracer tests.

The reference solution aims to capture all scales and provide a solution to the continuous equations, so that the tracer is resolved at all time (note that there is no molecular diffusion in the continuous equations). Therefore, the tracer variance of the reference solution should be conserved, as it is conserved in the continuous case. Any departure from conservation of tracer variance in the reference solution can be used to determine whether the reference solution is resolving all scales.

Another measure of numerical mixing is the entropy (Lauritzen and Thuburn, 2012)

$S_{q}=-k_{\mathrm{B}} \sum_{k=1}^{N} q_{k} \log q_{k} \rho_{k} \Delta A_{k}$,

where $k_{\mathrm{B}}$ is Boltzmann's constant, $N$ is the number of grid cells, and $\Delta A_{k}$ is the area of the grid cell. As the prescribed velocities in this document are non-divergent, we use $\rho$ equal to unity in the calculation of the entropy. To successfully model the downscale cascade to unresolved scales, the numerical schemes must dissipate tracer variance, and therefore create entropy. To determine how much entropy is generated by a numerical scheme we use the entropy diagnostic (Lauritzen and Thuburn, 2012)

$l_{\mathrm{s}}=\frac{S_{q}-S_{q}^{0}}{S_{q}^{0}}$.

Here, $S_{q}^{0}$ is the entropy of the initial conditions. For the reference solution, the tracer should be resolved for the duration of the simulation and there is no cascade to unresolved scales. Therefore, entropy should be conserved by the reference solution and $l_{\mathrm{s}}=0$ for all time.

In this paper, the reference solution will be calculated using CAM-FV with PPM and the default limiter with a $0.125^{\circ} \times 0.125^{\circ}$ grid spacing in the longitudinal and latitudinal directions. This corresponds to an equatorial grid spacing of about $14 \mathrm{~km}$. Using this scheme and resolution on the deformation test case 4 specified in Nair and Lauritzen (2010) gives a normalized $l_{2}$ error norm of 0.0005 for the Gaussian hills initial condition. This demonstrates that the scheme used to calculate the reference solutions is accurate. For the coarse resolution, we use a $2^{\circ} \times 2^{\circ}$ resolution $(91 \times 180$ grid points) for CAM-FV, a spectral triangular truncation of T85 with a $128 \times 256$ Gaussian transform grid for CAM-EUL, and ne16np4 resolution on the cubed-sphere grid for CAM-SE. The latter is composed of $16 \times 16$ grid cells on each cubed sphere face, with 4 internal node points, and is a similar resolution to $2^{\circ} \times 2^{\circ}$ on the latitude-longitude grid. CAM-SE is then interpolated onto the $2^{\circ} \times 2^{\circ}$ latitudelongitude grid using GECoRe. Note that for calculating error norms, the reference solution is averaged to the specific grid that each dynamical core uses (e.g. $2^{\circ} \times 2^{\circ}$ latitude-longitude grid for CAM-FV, the $128 \times 256$ Gaussian transform grid for CAM-EUL, and $2^{\circ} \times 2^{\circ}$ latitude-longitude for the GECoRe remapped CAM-SE). 

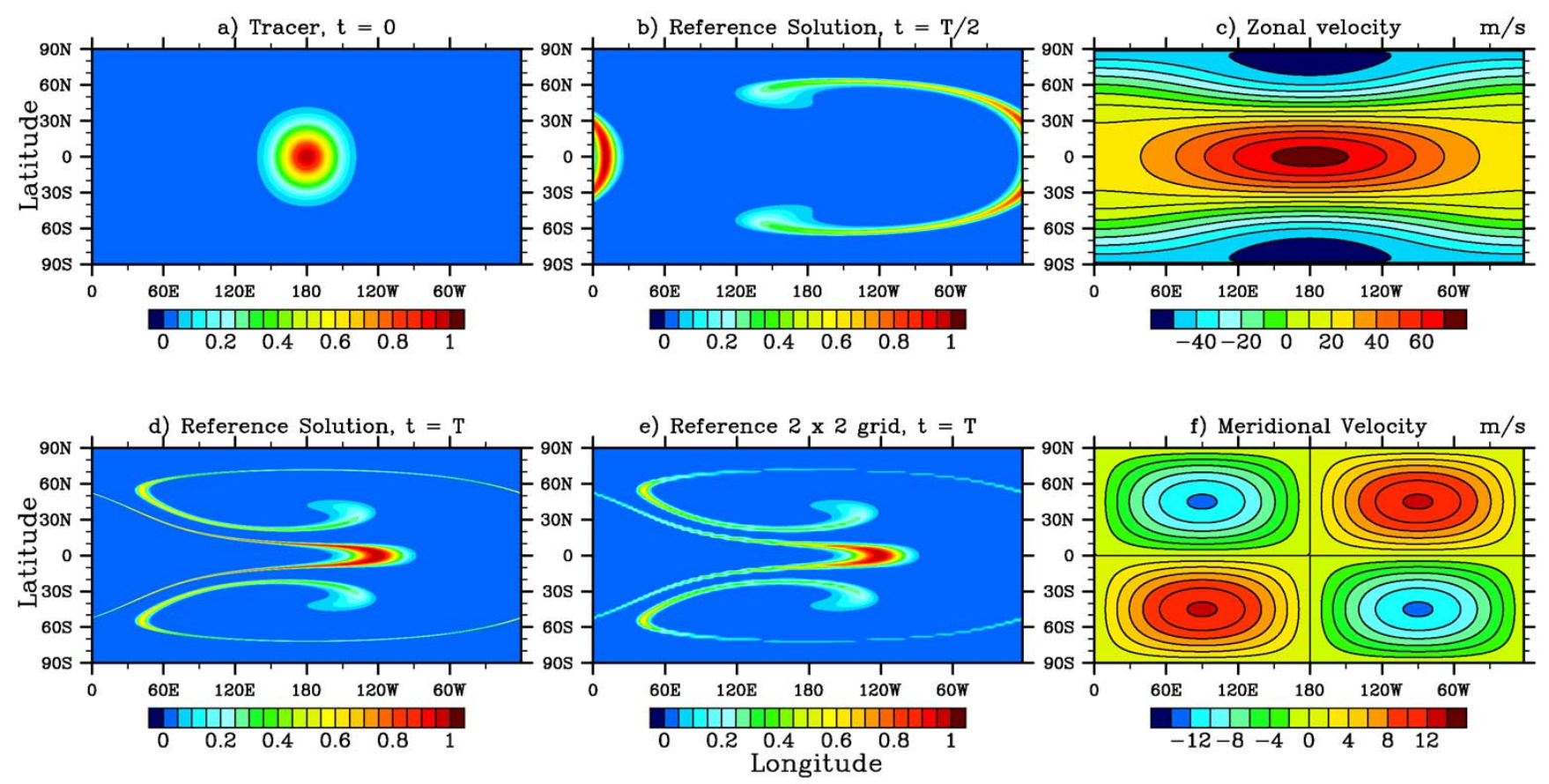

Fig. 1. Test 1 , (a) initial tracer, (b) the CAM-FV reference solution at $t=T / 2$ on the $0.125^{\circ} \times 0.125^{\circ}$ grid, (c) the zonal velocity $u$, (d) the reference solution at $t=T$, (e) the reference solution averaged onto the coarse $2^{\circ} \times 2^{\circ}$ resolution grid at time $t=T$, and (f) the meridional velocity, $v$.

\section{Two-dimensional test cases}

The aim of these tests is to determine how well a given advection scheme captures the downscale cascade of tracer variance and models the subgrid scales of the advection equation. Therefore, any explicit diffusion terms normally used with the advection scheme in a model, such as hyper-diffusion, is included when running the tests.

For non-divergent flow we can define the velocities in terms of the streamfunction $\psi$, where, on the sphere,

$$
\begin{aligned}
& u(\lambda, \phi)=-\frac{1}{R} \frac{\partial \psi}{\partial \phi}, \\
& v(\lambda, \phi)=\frac{1}{R \cos \phi} \frac{\partial \psi}{\partial \lambda},
\end{aligned}
$$

and $\phi$ is the latitude, $\lambda$ is the longitude and $R$ is the radius of the sphere.

\subsection{Small scale tests}

\section{Test 1}

The first test is designed to stretch the tracer below the grid scale of the coarse resolution grid. This test is designed so that a large part of the tracer is still resolved during the test, and this is advected around the equator. This test demonstrates the ability of the numerical schemes to model both resolved scale features and the cascade to unresolved scales on the same tracer. The streamfunction and velocities are given by

$$
\begin{aligned}
\psi(\lambda, \phi) & =\frac{1}{2} R K \sin (2 \phi) \\
& +\frac{1}{4} R K \cos (\lambda) \sin (2 \phi) \cos (\phi) \\
u(\lambda, \phi) & =K \cos (2 \phi) \\
& -\frac{1}{4} K \cos (\lambda)(2 \cos (2 \phi) \cos (\phi)-\sin (2 \phi) \sin (\phi)), \\
v(\lambda, \phi) & =-\frac{1}{4} K \sin (\lambda) \sin (2 \phi)
\end{aligned}
$$

with $K=8 R / T, R$ is the radius of the sphere and $T$ is the length of the simulation, in this case $T=12$ days. For the CAM intercomparison in this paper, we use $R=a$, where $a$ is the radius of the Earth. The initial tracer is a Gaussian hill, located at $(\pi, 0)$. The Gaussian hill is described by (Levy et al., 2007)

$q(\lambda, \phi)=\exp \left\{-r_{0}\left[\left(\tilde{X}-X_{\mathrm{c}}\right)^{2}+\left(\tilde{Y}-Y_{\mathrm{c}}\right)^{2}+\left(\tilde{Z}-Z_{\mathrm{c}}\right)^{2}\right]\right\}$,

where

$(\tilde{X}, \tilde{Y}, \tilde{Z})=(\cos \phi \cos \lambda, \cos \phi \sin \lambda, \sin \phi)$,

and $X_{\mathrm{c}}, Y_{\mathrm{c}}$ and $Z_{\mathrm{c}}$ are calculated using Eq. (17) and the tracer center $\left(\lambda_{c}, \phi_{c}\right)=(\pi, 0)$ in place of $\lambda$ and $\phi$. The dimensionless parameter $r_{0}=6$ determines the width of the 

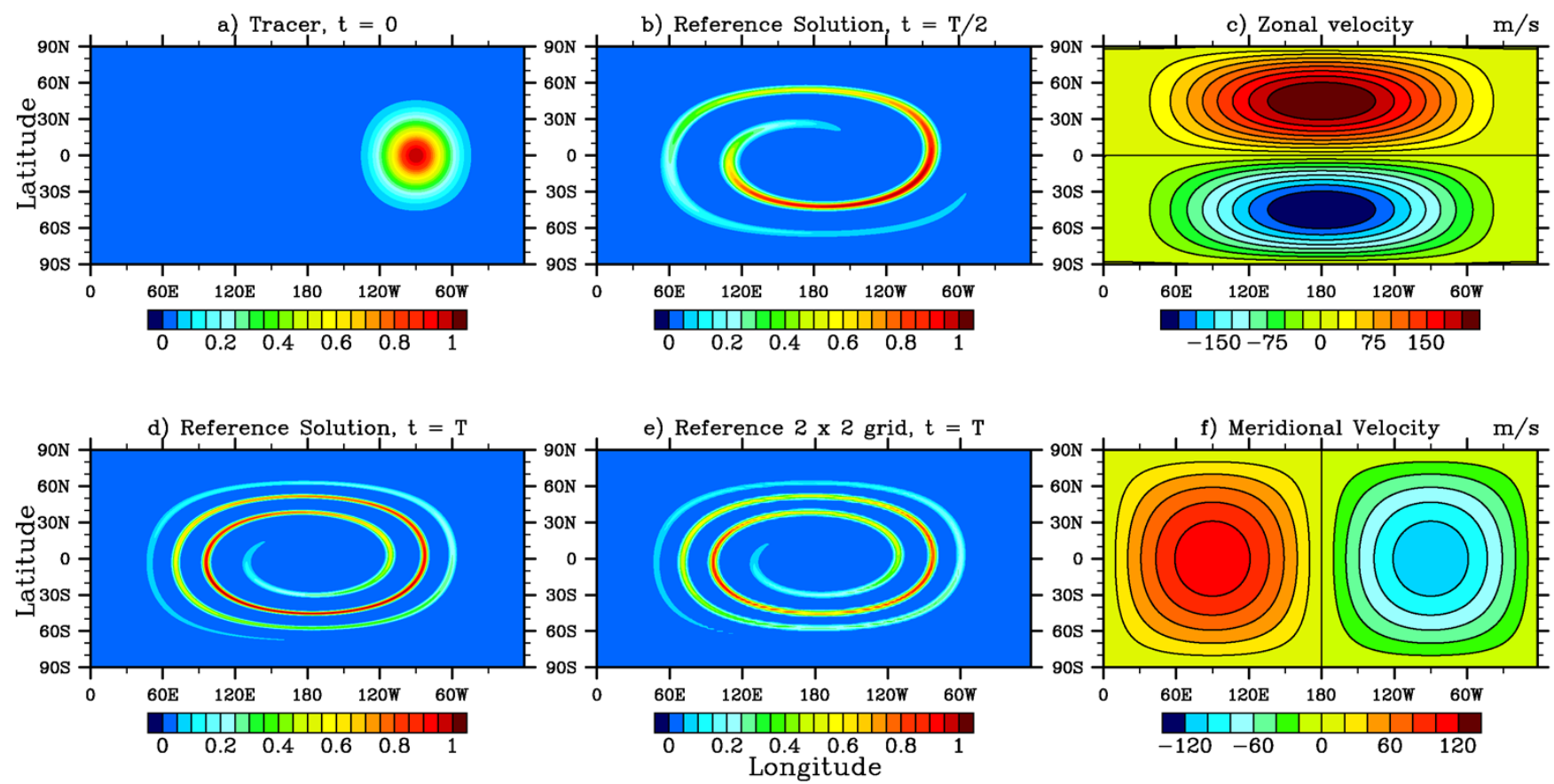

Fig. 2. As Fig. 1 but for test 2.

Gaussian hill. Figure 1 shows the initial tracer, the CAMFV reference solution, the reference solution averaged onto the coarse $2^{\circ} \times 2^{\circ}$ resolution grid and the velocities for test 1 . A time step of $30 \mathrm{~s}$ is used to calculate the reference solution for each test.

\section{Test 2}

The second test is an extension of the first deformation test from Nair and Lauritzen (2010); the time dependent term is removed, and the tracer is stretched out in a spiral. After some time, the tracer will be stretched below the grid scale of the coarse grid. The streamfunction and velocities are given by

$$
\begin{aligned}
\psi(\lambda, \phi) & =R K \sin ^{2}(\lambda / 2) \cos ^{2}(\phi), \\
u(\lambda, \phi) & =K \sin ^{2}(\lambda / 2) \sin (2 \phi), \\
v(\lambda, \phi) & =\frac{K}{2} \sin (\lambda) \cos (\phi),
\end{aligned}
$$

with $K=38 R / T$, and $T=12$ days. The initial tracer is a Gaussian hill, located at $\left(\lambda_{\mathrm{c}}, \phi_{\mathrm{c}}\right)=(3 \pi / 2,0)$ with $r_{0}=5$ in Eq. (16). The initial tracer, the reference solution using $\mathrm{CAM}-\mathrm{FV}$ on the $0.125^{\circ} \times 0.125^{\circ}$ grid, the reference solution averaged onto the coarse grid of $2^{\circ} \times 2^{\circ}$ resolution, and the velocities are shown in Fig. 2 for test 2.

\section{Test 3}

The third test is designed to challenge the advection schemes' ability to handle small scale tracer filaments as they are transported across the poles. This is important because some errors when solving advection problems on the sphere, especially on a latitude-longitude grid, are due to the pole points. The test is similar in design to the moving vortex problem proposed by Nair and Jablonowski (2008); it is a mix between polar vortices and a solid body rotation over the poles. The streamfunction and velocities are given as

$$
\begin{aligned}
\psi(\lambda, \phi) & =\frac{R K}{2} \cos (2 \phi)-R U_{0} \cos (\phi) \cos (\lambda-\pi), \\
u(\lambda, \phi) & =K \sin (2 \phi)-U_{0} \sin (\phi) \cos (\lambda-\pi), \\
v(\lambda, \phi) & =U_{0} \sin (\lambda-\pi),
\end{aligned}
$$

with $K=10 R / T$ and $U_{0}=4 \pi R / T$. This test is also run for $T=12$ days. The initial tracer is a Gaussian hill, centered at $\left(\lambda_{\mathrm{c}}, \phi_{\mathrm{c}}\right)=(3 \pi / 2, \pi / 4)$, with $r_{0}=10$ in Eq. (16). The initial tracer, the CAM-FV reference solution, the reference solution averaged onto the coarse $2^{\circ} \times 2^{\circ}$ resolution grid and the velocities are shown in Fig. 3 for test 3 .

\subsection{Separate cells test}

\section{Test 4}

To test whether the diffusion in the tracer transport scheme of a dynamical core shows unphysical characteristics, the final two-dimensional test is designed such that there are two flow cells separated by a barrier. The tracer is initialized in the western hemisphere, and due to the analytically prescribed velocities no mass should move into the eastern hemisphere. The amount of mass in the eastern hemisphere at the end of 

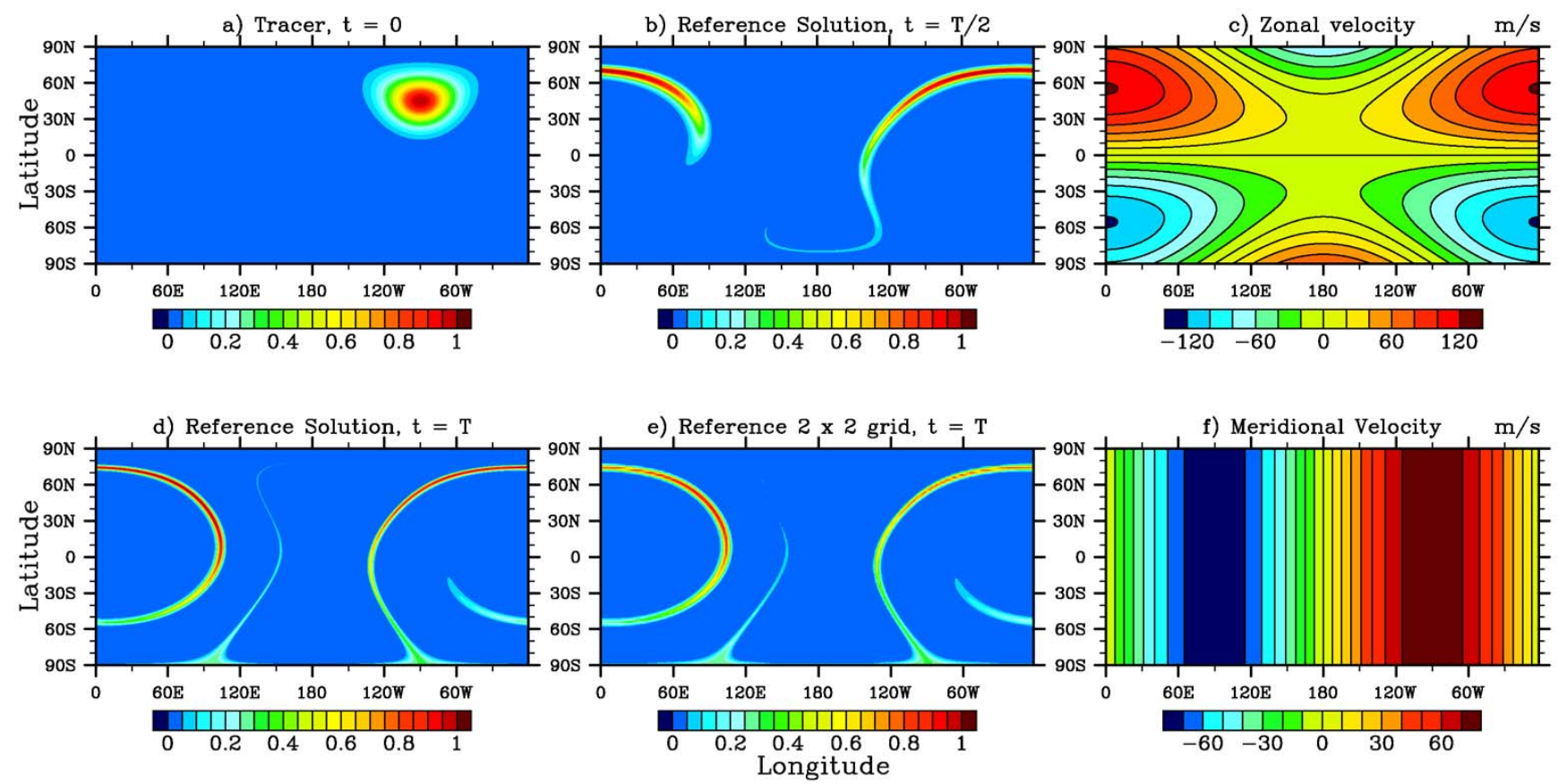

Fig. 3. As Fig. 1 but for test 3 .

the test will show whether the characteristics of the numerical scheme are unphysical.

The initial velocities for the separate cells test are from test case 2 from Nair and Lauritzen (2010) without the time dependent terms, and are given as

$$
\begin{aligned}
\psi(\lambda, \phi) & =R K \sin ^{2}(\lambda) \cos ^{2}(\phi), \\
u(\lambda, \phi) & =K \sin ^{2}(\lambda) \sin (2 \phi), \\
v(\lambda, \phi) & =K \sin (2 \lambda) \cos (\phi) .
\end{aligned}
$$

The magnitude of the velocity is $K=16 R / T$, and the simulation is run for $T=24$ days. There are two sets of initial tracers; cosine bells and slotted cylinders. The two cosine bells are centered at $\left(\lambda_{c 1}, \phi_{c 1}\right)=(17 \pi / 96, \pi / 8)$ and $\left(\lambda_{c 2}, \phi_{c 2}\right)=(79 \pi / 96,-\pi / 8)$. The cosine bell is defined as

$q(\lambda, \phi)= \begin{cases}h & \text { if } \quad r \leq d, \\ 0 & \text { otherwise }\end{cases}$

where

$h=\frac{1}{2}[1+\cos (\pi r / d)]$,

$d=0.5$, and $r_{i}$ with $i=1,2$ denotes the great circle distance of a unit sphere

$r_{i}=\arccos \left(\sin \phi_{c i} \sin \phi+\cos \phi_{c i} \cos \phi \cos \left(\lambda-\lambda_{c i}\right)\right)$.
The slotted cylinders are centered at the same points as the cosine bells, and are defined as

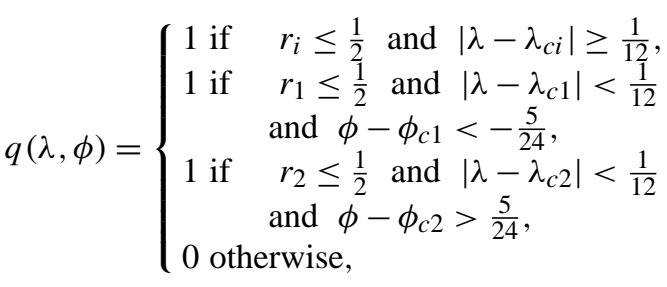

The position of the barrier is along the longitude $\lambda=\pi$. To change the position of the dividing barrier, we can replace Eq. (24) with

$$
\psi(\lambda, \phi)=K \sin ^{2}\left(\lambda-\lambda_{\mathrm{B}}\right) \cos ^{2}(\phi),
$$

where $\lambda_{B}$ is the deviation of the barrier from $\lambda=\pi$. The tracer centers become $\left(\lambda_{c}, \phi_{c}\right)=\left(17 \pi / 96+\lambda_{B}, \pi / 8\right)$ and $\left(79 \pi / 96+\lambda_{\mathrm{B}},-\pi / 8\right)$.

\subsection{Numerical effect of the subgrid terms}

Using the tests described above, we can numerically investigate the effect of the subgrid terms of the discrete advection Eq. (6) on tracer variance. On a high-resolution grid we run the above tests, and at 6 hour intervals we average onto coarser resolution grids (i.e. $1 / 2^{\circ} \times 1 / 2^{\circ}, 1^{\circ} \times 1^{\circ}, 2^{\circ} \times 2^{\circ}$ and $4^{\circ} \times 4^{\circ}$ ). For each coarse resolution grid, we calculate the normalized tracer variance of the reference solution and plot it as a time series. The normalized tracer variance is calculated by dividing Eq. (8) by $\int_{A} \bar{\rho} d A$. The reference solution 

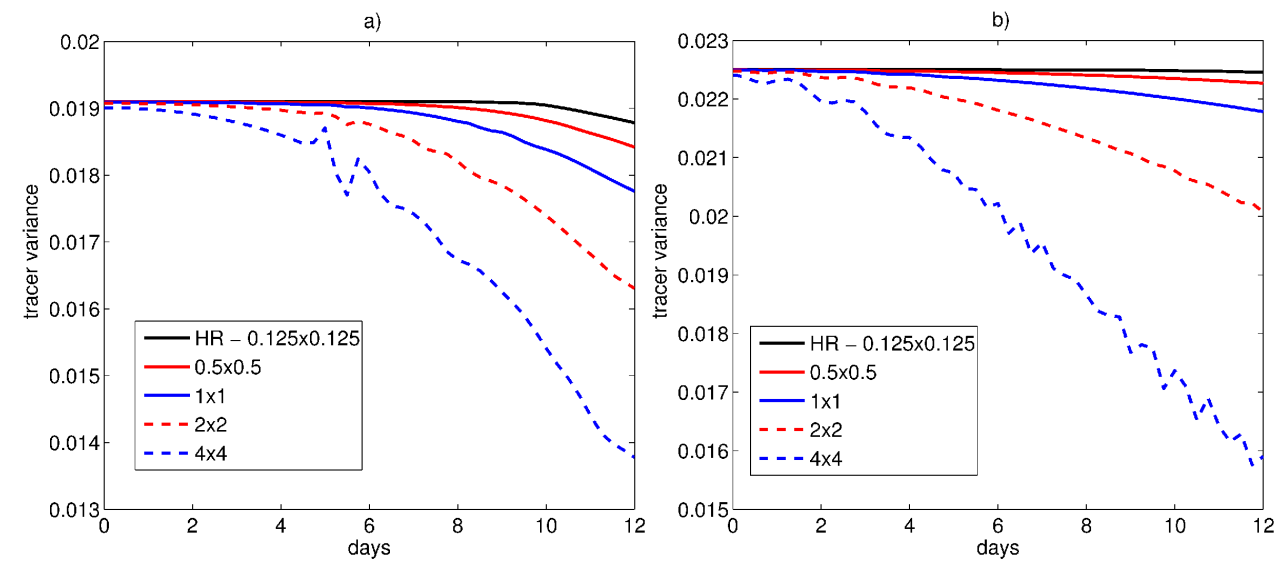

Fig. 4. Normalized tracer variance against time for the CAM-FV high resolution (HR) reference solution, and the reference solution averaged onto the coarse grids, for (a) test 1 , and (b) test 2.
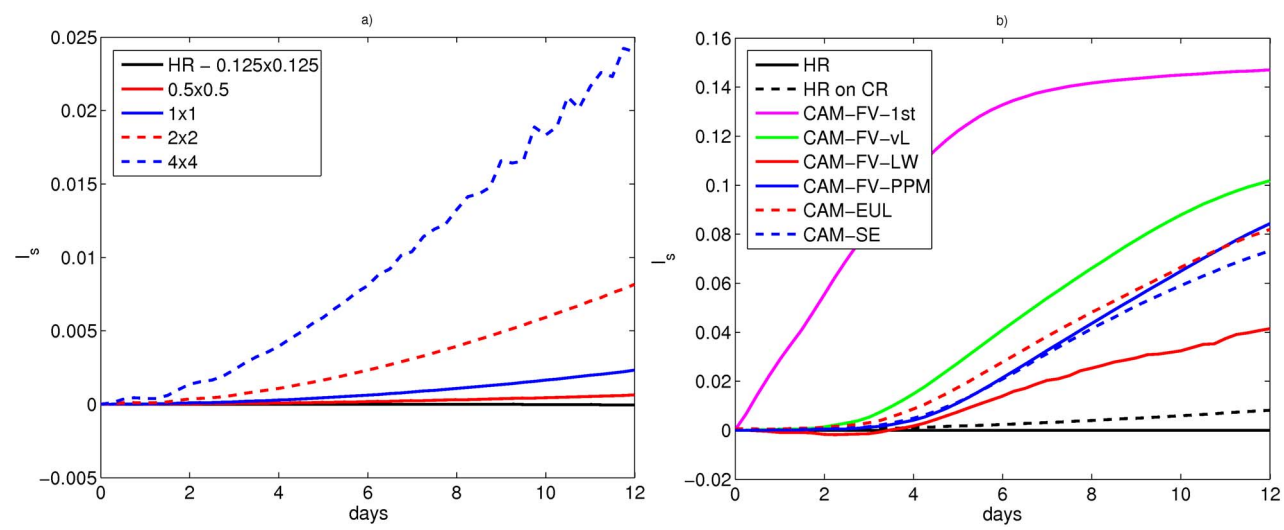

Fig. 5. Entropy diagnostic $l_{\mathrm{S}}$ against time for test 2 for (a) the CAM-FV reference solution, and the reference solution averaged onto coarser grids, and (b) the (HR) CAM-FV reference solution, (CR) reference solution averaged onto the coarse grid, CAM-FV at $2^{\circ} \times 2^{\circ}$ resolution with the (1st) 1st-order, (vL) van Leer, (LW) Lax-Wendroff, and PPM with the default limiter schemes, CAM-EUL at T85 resolution and CAM-SE. Note the different scales on the y-axis.

contains the effects of scales that cannot be resolved on the coarser grids, and will show the effects of the subgrid scales on tracer variance.

The normalized tracer variance is plotted against time in Fig. 4 for tests 1 and 2. The tracer variance for the reference solution and the reference solution averaged onto the coarse grids are shown. The reference solution almost conserves tracer variance. Averaging the reference solution to the coarse grids provides a solution that contains the effects of scales smaller than the grid, i.e. equivalent to solving Eq. (6) exactly. The amount of tracer variance decreases with time, for both tests, when the solution is averaged onto the coarse $1 / 2^{\circ} \times 1 / 2^{\circ}, 1^{\circ} \times 1^{\circ}, 2^{\circ} \times 2^{\circ}$ and $4^{\circ} \times 4^{\circ}$ grids. This shows that tracer variance is not conserved in the discrete equations, and that it must cascade downscale to scales that are not resolved on the coarse grids.
The left plot of Fig. 5 shows the entropy diagnostic, $l_{\mathrm{s}}$, plotted against time for the reference solution and the reference solution averaged onto the coarse grids for test 2 . For the reference solution $l_{\mathrm{s}}=0$ for all time. As the reference solution is averaged onto the coarser grid $l_{\mathrm{s}}$ increases with time, showing that entropy is being produced. This shows that an effect of the cascade to unresolved scales is the production of entropy.

\section{Results using CAM}

The final tracer mixing ratios when using the schemes described in Sect. 2 , on the $2^{\circ} \times 2^{\circ}$ resolution grid for CAM$\mathrm{FV}$, T85 resolution for CAM-EUL and ne16np4 resolution for CAM-SE, are shown in Fig. 6 for test 1. The tracer mixing ratios are shown at time $t=T$ and can be compared with the reference solution averaged onto the coarse resolution grid 


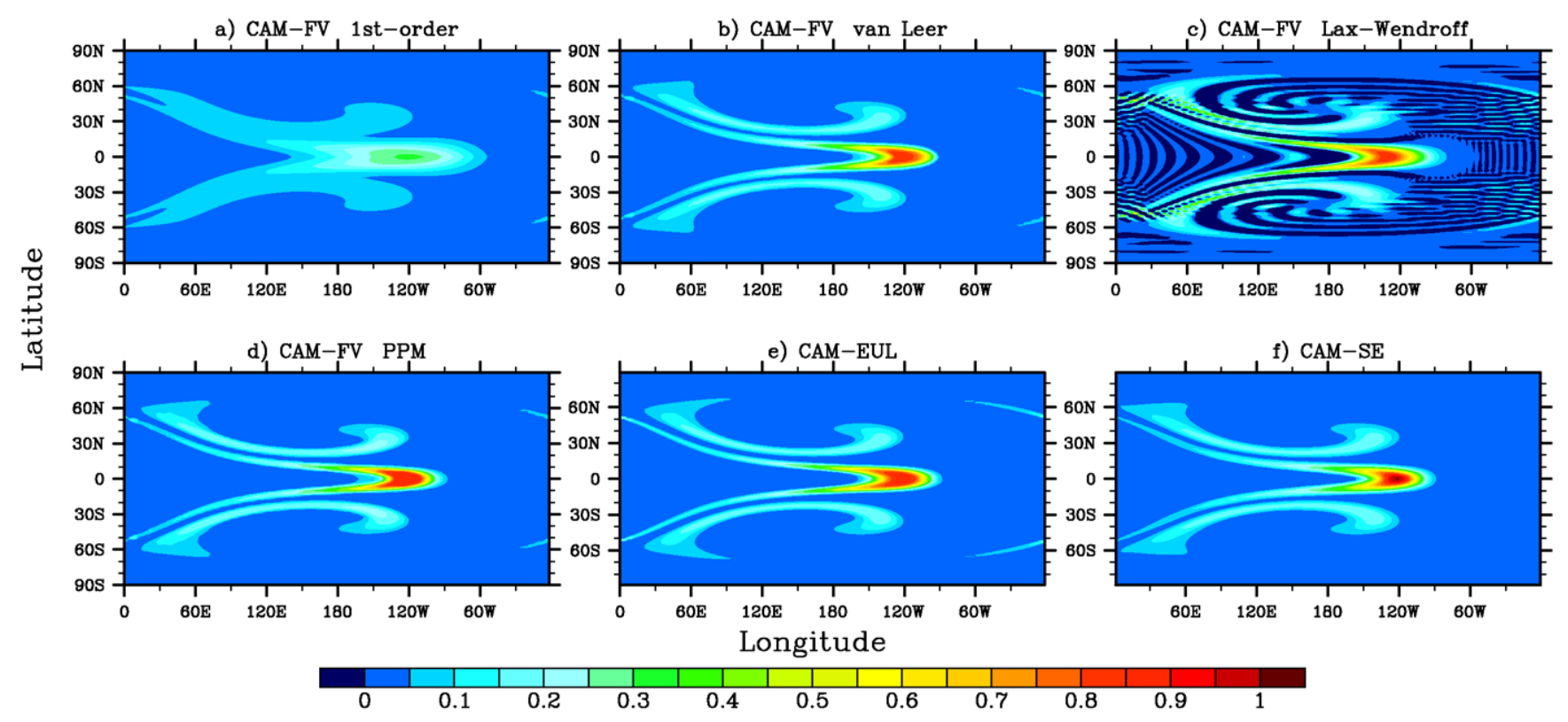

Fig. 6. Tracer mixing ratio results using CAM for test 1 at time $t=T$. CAM-FV using (a) first-order, (b) van-Leer, (c) Lax-Wendroff and (d) PPM with the default limiter, and (e) CAM-EUL, and (f) CAM-SE. These plots can be compared with the reference solution averaged onto the coarse resolution grid in Fig. 1. The maximum tracer mixing ratio values are (a) 0.311 , (b) 0.832 , (c) 0.854 , (d) 0.886 , (e) 0.865 , and (f) 1.00 . Note that the maximum of the CAM-FV reference solution averaged onto the $2^{\circ} \times 2^{\circ}$ resolution grid is 0.9974 .

shown in Fig. 1. For this test we also show the results for the different numerical fluxes in CAM-FV; first-order, vanLeer and Lax-Wendroff. Although these numerical methods would not be used operationally, the results highlight the effects of both too much and too little diffusion.

For CAM-FV, the first-order scheme has over diffused the tracer, and the thin tracer filaments have been merged into one. The Lax-Wendroff scheme has produced dispersion errors that propagate throughout the domain and cause negative undershoots. Neither of these schemes are able to capture the subgrid term and correctly model the cascade to unresolved scales. The van Leer scheme is less diffusive than first-order, but it has diffused more of the tracer mass in the center of the domain than CAM-FV with PPM and the default limiter. The solutions for CAM-FV with PPM and the default limiter, CAM-EUL and CAM-SE are very similar. They have each successfully reproduced the large scale tracer mass in the center of the domain, although each scheme has also smoothed out the stretched tracer filaments. CAM-SE has the largest maximum and steepest gradients of the tracer mass in the center of the domain, with the maximum value exceeding that of the reference solution. This overshoot occurs in CAM-SE because the limiter chosen in this test is only positive definite, and does not guarantee monotonicity.

Figure 7 shows the results for test 2. Each of the schemes spreads out the tracer more than the reference solution. CAM-EUL has begun to merge two of the filaments into one (note that CAM-EUL is tested with a slightly finer grid spacing of about $156 \mathrm{~km}$ and therefore can resolve more of the tracer than CAM-FV and CAM-SE). Both CAM-SE and CAM-FV keep the filaments of the tracer spiral more distinct. All three schemes diffuse the tail end of the tracer. CAM-SE has the largest mixing ratio maximum with a value of 0.368 , compared to 0.301 for CAM-FV and 0.259 for CAM-EUL.

Test 3, shown in Fig. 8, is designed to challenge the advection algorithm for cross-polar flow, and this is relevant to schemes designed on latitude-longitude grids (e.g. CAMFV). As with tests 1 and 2, CAM-EUL is the most diffusive of the CAM dynamical cores. No dynamical core experiences significant problems while modeling the stretched out tracer as it passes over the poles.

At each time step the normalized $l_{2}$ error norms can be calculated for the mixing ratios $q$. The high resolution reference solution is averaged onto the coarse grid used by the dynamical cores (e.g. $2^{\circ} \times 2^{\circ}$ for CAM-FV and the GECoRe remapped CAM-SE, and the $128 \times 256$ Gaussian transform grid for CAM-EUL), and this is used as the reference solution on the coarse grid, $q_{\mathrm{C}}$. The normalized $l_{2}$ error norm is then calculated as

$l_{2}=\left[\frac{I\left[\left(q-q_{\mathrm{C}}\right)^{2}\right]}{I\left[\left(q_{\mathrm{C}}\right)^{2}\right]}\right]^{\frac{1}{2}}$,

where $I$ is the global two-dimensional integral (as in Williamson et al., 1992).

The normalized $l_{2}$ error norms for tests 1 and 2 are plotted against time in Fig. 9. For test 1 we include the error norms of the different numerical fluxes in CAM-FV. The 


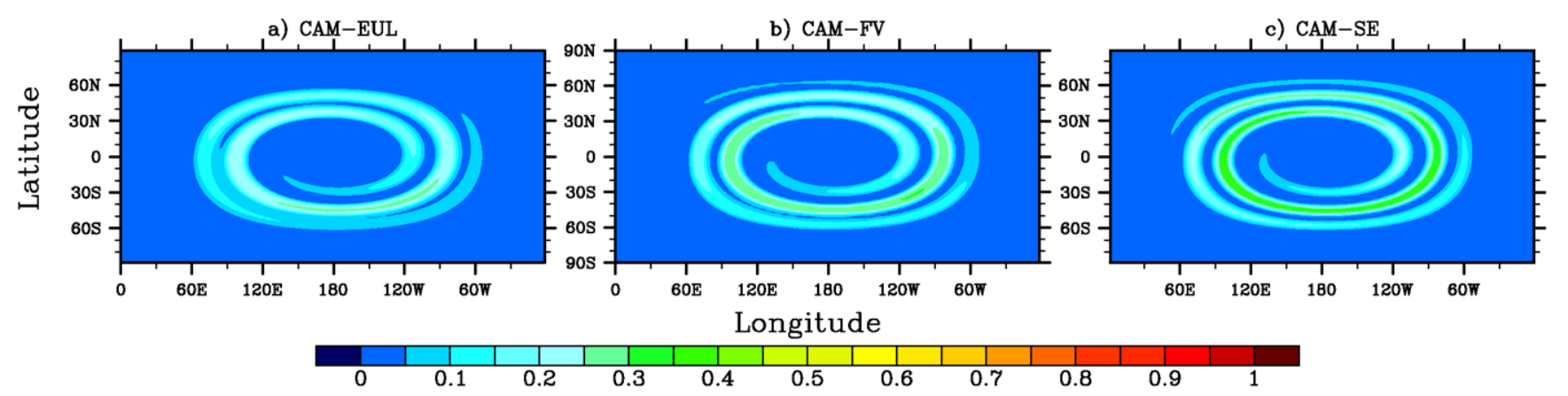

Fig. 7. Tracer mixing ratio results using, (a) CAM-EUL, (b) CAM-FV (PPM) and (c) CAM-SE for test 2 at time $t=T$. These plots can be compared with the reference solution averaged onto the coarse resolution grid in Fig. 2.

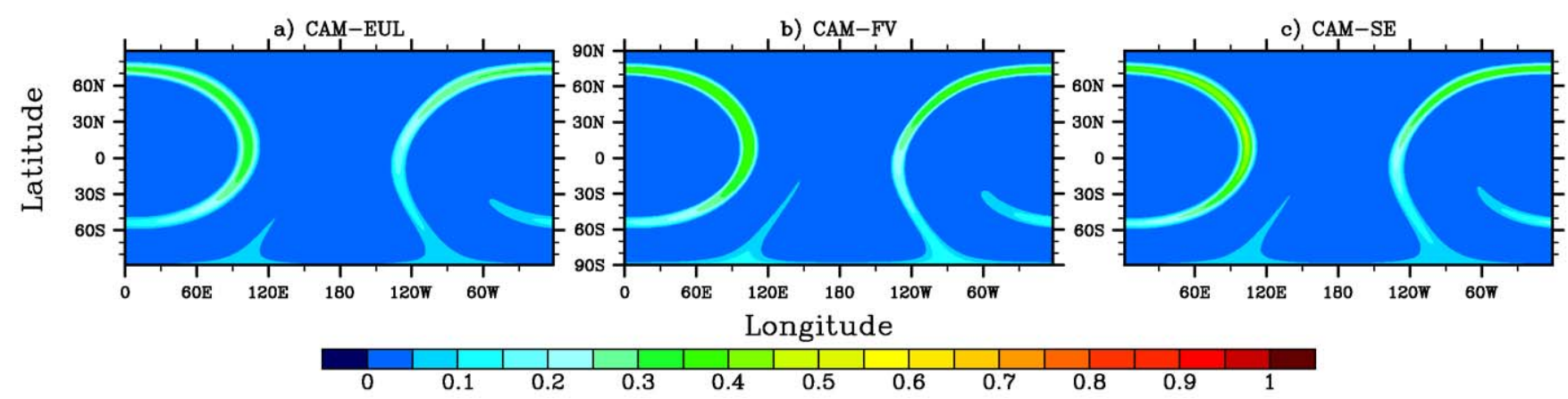

Fig. 8. Tracer mixing ratio results using (a) CAM-EUL, (b) CAM-FV and (c) CAM-SE for test 3 at time $t=T$. These plots can be compared with the reference solution averaged onto the coarse resolution grid in Fig. 3.

van Leer scheme and PPM with the default limiter have the smallest error norms for CAM-FV. The largest error norms are for the first-order scheme and Lax-Wendroff. This is because the first-order scheme is very diffusive, and the Lax-Wendroff scheme is predominantly dispersive. Neither scheme accurately models the downscale cascade. The firstorder scheme diffuses both large and small scales, whereas the Lax-Wendroff scheme does not prevent the build up of grid scale noise (it amplifies the grid scale features instead of diffusing them). Out of the operational dynamical cores, CAM-EUL produces the largest error norms, even though CAM-EUL is tested on a higher resolution grid than CAMFV and CAM-SE. CAM-SE has the smallest error norm for test 2, however, during test 1 the error rises above that of CAM-FV. This may be due to the overshoots producing the large maximum found in the tracer mass in the center of the domain.

Figure 10 shows the normalized tracer variance against time for each scheme for tests 1 and 2. Also shown are the normalized tracer variance statistics for the CAM-FV reference solution, and for the reference solution averaged onto the $2^{\circ} \times 2^{\circ}$ grid. For each test case, the reference solution almost conserves tracer variance. This shows that the tests are almost completely "resolved" on the $1 / 8^{\circ} \times 1 / 8^{\circ}$ grid, and, when considering the error norm of the reference solution for the deformation test (Nair and Lauritzen, 2010), that the solution is accurate. The averaged reference solution shows a decrease in tracer variance with time. This indicates that tracer variance is being transferred to scales that cannot be resolved on the $2^{\circ} \times 2^{\circ}$ grid; i.e. a downscale cascade of tracer variance.

Again, we include the results for the different numerical fluxes in CAM-FV for test 1. The diffusion in the first-order scheme is evident, as the tracer variance drops off steeply and approaches zero. The van Leer scheme is the next most diffusive scheme. The Lax-Wendroff scheme does not dissipate tracer variance, and therefore the tracer variance is greater than that of the reference solution averaged onto the coarse grid. For the dynamical cores, CAM-EUL dissipates the most tracer variance. CAM-SE has good tracer variance statistics for test 2, but as with the error norms it performs worse for test 1 . The tracer variance for each of these schemes is less than that of the reference solution averaged onto the coarse grid. This implies that each of the schemes are dissipating too much tracer variance. For test 3 both the error norms and the normalized tracer variance for CAM-FV, CAM-EUL and CAM-SE are similar to the results from test 2 (and are therefore not shown). For example, the normalized $l_{2}$ error norms at day 12 are $0.541,0.625$ and 0.498 , for CAM-FV, CAM-EUL and CAM-SE, respectively. This indicates that 

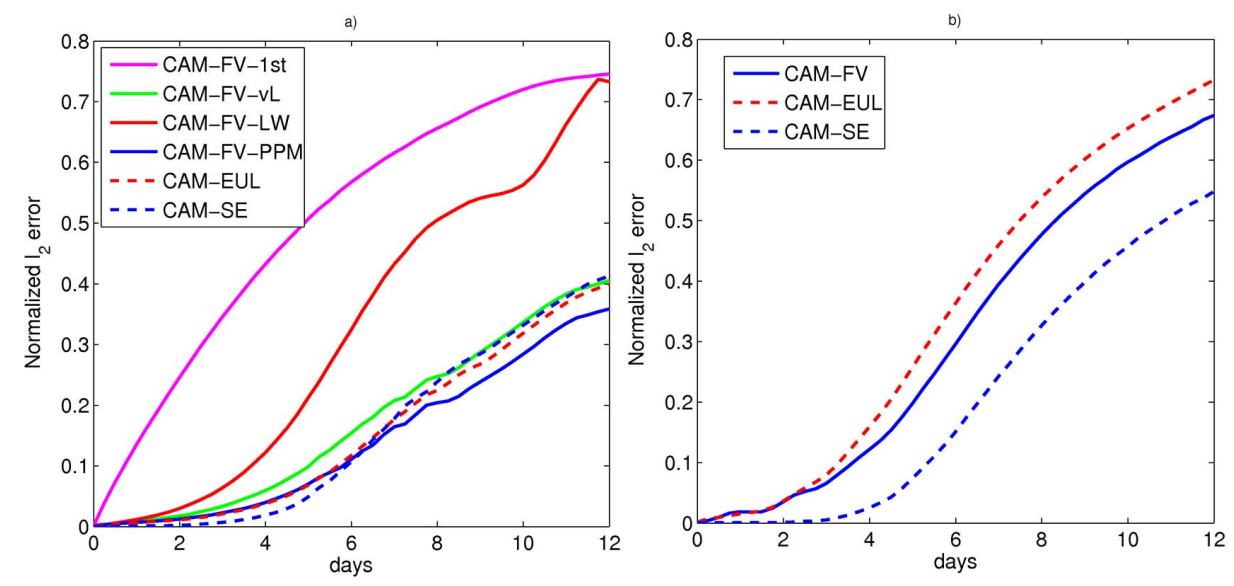

Fig. 9. Normalized $l_{2}$ error norms against time for (a) CAM-FV at $2^{\circ} \times 2^{\circ}$ resolution with the (1st) 1 st-order, (vL) van Leer, (LW) LaxWendroff, and PPM with the default limiter schemes, CAM-EUL at T85 resolution and CAM-SE for test 1, and for (b) the default versions of CAM-FV, CAM-EUL and CAM-SE for test 2.
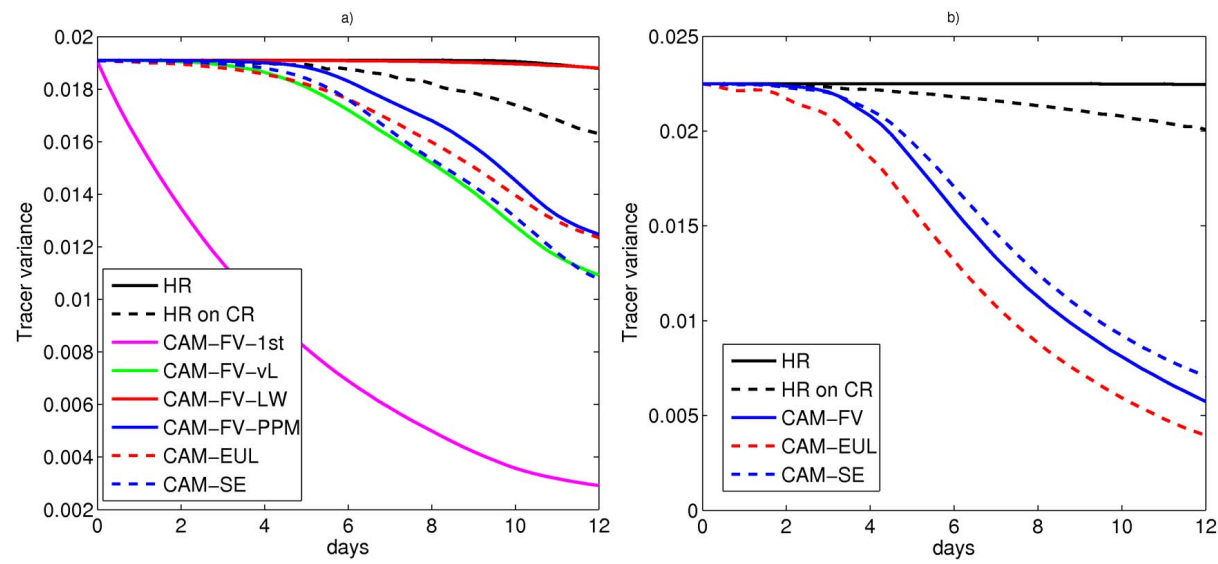

Fig. 10. Normalized tracer variance against time for (a) the (HR) CAM-FV reference solution, (CR) reference solution averaged onto the coarse grid, CAM-FV at $2^{\circ} \times 2^{\circ}$ resolution with the (1st) 1 st-order, (vL) van Leer, (LW) Lax-Wendroff, and PPM with the default limiter schemes, CAM-EUL at T85 resolution and CAM-SE, for test 1, and for (b) the (HR) CAM-FV reference solution, (CR) reference solution averaged onto the coarse grid, and the default versions of CAM-FV, CAM-EUL and CAM-SE for test 2.

the cross-polar component does not affect the accuracy of the advection schemes in the CAM dynamical cores.

The entropy diagnostic for each scheme for test 2 is plotted against time in the right plot of Fig. 5. These results agree with those from the tracer variance statistics. The first-order scheme creates too much entropy due to the excessive implicit diffusion in the scheme. The Lax-Wendroff scheme produces the least amount of entropy of the schemes, and at $t \approx 2$ days has negative values for $l_{\mathrm{s}}$. This means that the amount of entropy is decreasing at this time, due to the dispersion errors in the Lax-Wendroff scheme dominating any numerical mixing. CAM-FV with the van Leer scheme and with PPM, and CAM-EUL and CAM-SE all produce more entropy than the reference solution averaged onto the coarse grid. The entropy diagnostics for tests 1 and 3 produce almost identical results.

For test 4 there is no need to compare with a highresolution reference solution. Physically, there should be no mass in the eastern hemisphere. Any mass in the eastern hemisphere in the numerical simulations must be due to numerical error, such as dispersion errors propagating across the divide, excessive diffusion that spreads the tracer across the divide or errors due to the position of the grid cells compared to the dividing barrier. We use each of the numerical fluxes for CAM-FV. Using the cosine bell initial tracers after 24 days, the Lax-Wendroff scheme again produces dispersion errors that have propagated across the whole domain. The other schemes are all diffusive, and they have spread out the tracers as they are stretched and deformed; the individual 


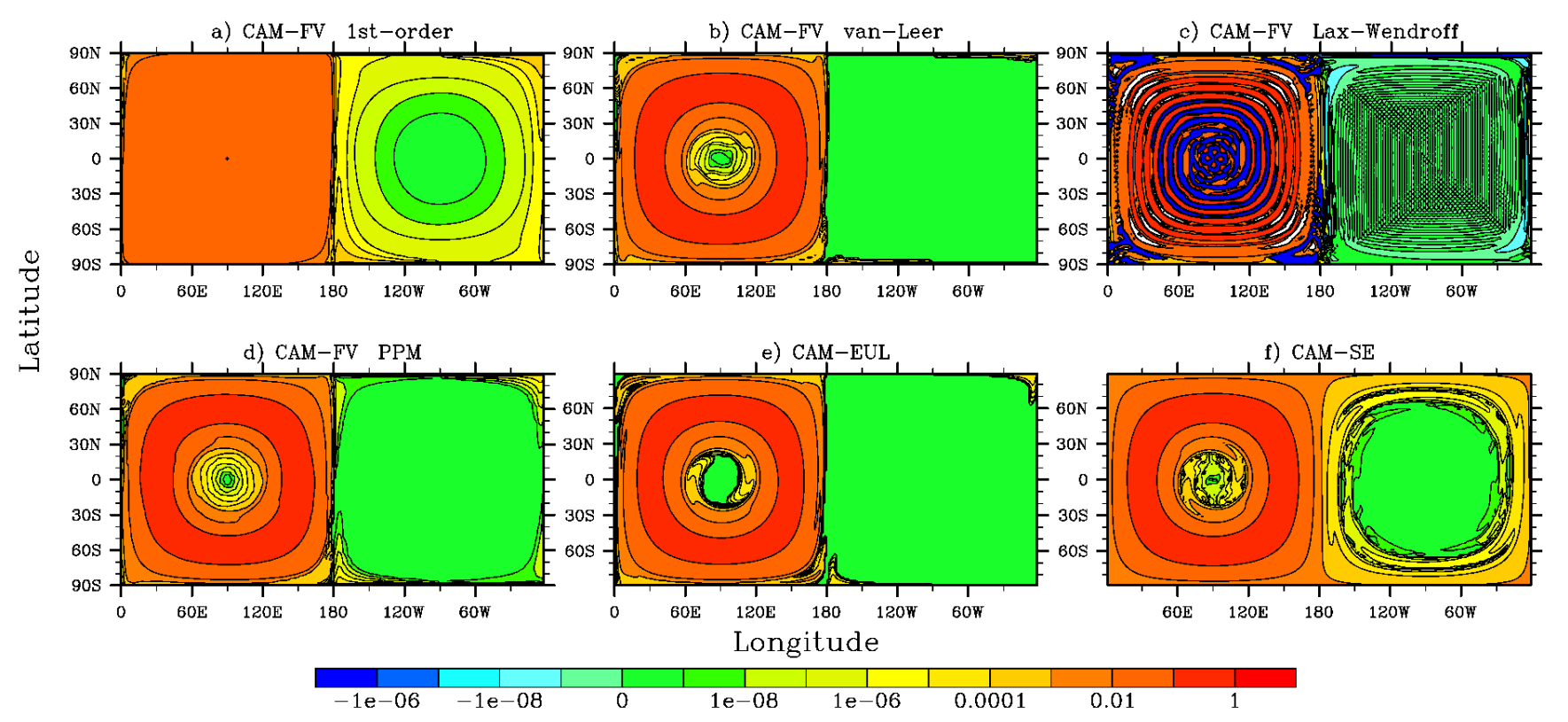

Fig. 11. Tracer mixing ratio results for Test 4. At time $t=T$, CAM-FV (a) 1st-order, (b) van Leer, (c) Lax-Wendroff, and (d) PPM with the default limiter, (e) CAM-EUL and (f) CAM-SE. The contours use a log scale.

Table 1. Percentage of mass in the eastern hemisphere for test 4.

\begin{tabular}{lcr}
\hline & Cosine Bell & Slotted Cylinder \\
\hline First-Order & $9.03 \times 10^{-3}$ & $3.04 \times 10^{-2}$ \\
van Leer & $5.79 \times 10^{-5}$ & $6.37 \times 10^{-4}$ \\
Lax-Wendroff & $1.53 \times 10^{-4}$ & $3.10 \times 10^{-2}$ \\
CAM-FV (PPM) & $5.43 \times 10^{-5}$ & $1.03 \times 10^{-3}$ \\
CAM-EUL & $3.80 \times 10^{-5}$ & $1.69 \times 10^{-3}$ \\
CAM-SE & $1.21 \times 10^{-3}$ & $1.67 \times 10^{-2}$ \\
\hline
\end{tabular}

tracer filaments have all been smoothed into one "ring". The results using the slotted cylinder tracers are similar. Figure 11 shows the tracer mixing ratios at day 24 for the cosine bell initial conditions, using a $\log$ scale for the contours. This quite clearly shows that each of the schemes have allowed mass to move into the eastern hemisphere. Much of the mass actually passes across the poles. For CAM-FV on $2^{\circ} \times 2^{\circ}$ resolution, the dividing barrier lies across the center point of the grid cells at $\lambda=\pi$. This means that any mass that falls into these grid cells in the western hemisphere will be represented as being in both hemispheres due to the nature of the finite-volume grid cell on the latitude-longitude grid. Similarly, for the cubed sphere grid used with CAM-SE, the dividing barrier is not aligned with the edges of the cubed sphere grid cells. As the grid cells near the poles are much larger than on the corresponding latitude-longitude grid, this allows more mass to be passed from west to east. This results in a large amount of mass in the eastern hemisphere when using CAM-SE.
We calculate the percentage of the tracer mass that is in the eastern hemisphere. As the Lax-Wendroff scheme produces negative values, we use the absolute value of the tracer mixing ratio. The results for the cosine bells and the slotted cylinder initial conditions are given in Table 1. Although the values are much less than $1 \%$, physically they should be exactly zero. The first-order scheme has the largest values, due to the excessive diffusion spreading the tracer across the divide. The Lax-Wendroff scheme also has large values, and this is due to the dispersive nature of the scheme. CAM-SE has also produced large amounts of mass in the eastern hemisphere, however, as stated above, this is mainly due to the cubed sphere grid. The van Leer scheme, CAMFV with PPM with the default limiter and CAM-EUL have much smaller values. Even so, the fact that they are greater than zero shows that at some time up to day 24 , the diffusion inherent in these schemes violates the barrier between the eastern and western hemispheres.

\section{Conclusions}

Tracer advection algorithms need challenging test cases to show how well they perform when there is a downscale cascade of tracer variance, a process that needs to be accurately modelled in dynamical cores of weather and climate models. We have extended the existing literature to develop three test cases that stretch the tracers below the grid scale and generate a downscale cascade. High-resolution simulations provide a reference solution that can numerically show the effect of these subgrid terms, and the downscale cascade of tracer 
variance. Some form of diffusion is required by the numerical schemes to act as a subgrid model, prevent noise at the grid scale, and correctly model the downscale tracer variance cascade. We have designed a fourth test case that highlights some of the unphysical characteristics of diffusion in tracer advection algorithms. Two separate flow cells are initialized so that no mass should cross the barrier between the cells. Any tracer mass that passes the barrier is due to unphysical numerical error.

We use these tests to determine the accuracy of the horizontal tracer transport algorithms in three dynamical cores of NCAR's CAM version 5: CAM-FV, CAM-EUL and CAMSE. We use error norms, normalized tracer variance statistics and entropy diagnostics as methods of comparison. A number of different flux operators are used with the LinRood method in CAM-FV to highlight different characteristics of advection schemes. The first-order scheme always over-diffuses the tracers, and this shows that too much diffusion does not model the small scales accurately and can destroy large scale features of the flow. The Lax-Wendroff scheme is predominantly dispersive and could not capture the cascade to unresolved scales because there is no dominant diffusive mechanism to dissipate tracer variance. For some tests the Lax-Wendroff scheme appears to have good tracer variance statistics and entropy diagnostics. However, the corresponding error norms show that the Lax-Wendroff scheme actually performs badly, and this shows that we should use a range of measurements to validate the accuracy of a scheme. The van Leer scheme performs well, but is always less accurate than the default option in CAM-FV (PPM and the default limiter). The operational CAM-FV performs well, and captures some of the downscale tracer variance cascade through the implicit diffusion due to the limiter in PPM. The mixture of limiters and hyper-diffusion in CAM-SE produces good tracer variance statistics, and a solution without noise. However, as CAM-SE uses a "positive-definite" constraint overshoots can occur, and this reduces the accuracy for test 1 . In their default configurations both CAM-FV and CAM-SE outperform CAM-EUL. This is due to the semi-Lagrangian scheme in CAM-EUL being more diffusive than the tracer transport schemes in CAM-FV and CAM-SE. For each test, all three of the CAM dynamical cores dissipates more tracer variance than the reference solution averaged onto the corresponding coarse grid.

All of the schemes produce tracer mass that crosses a prescribed barrier when performing the separate flow cells test. This is due to dispersion errors (in the Lax-Wendroff scheme), diffusion spreading the tracer across the divide, and also the alignment of grid cells with the barrier. This shows that although CAM-FV, CAM-EUL and CAM-SE were able to model the downscale tracer variance cascade, the type of diffusion in these schemes (both implicit and explicit) violates the barrier between the two flow cells, and in that sense becomes unphysical at some point before day 24 of the separate flow cells test.
Acknowledgements. We would like to thank two anonymous reviewers for their helpful comments. We would also like to thank Paul Ullrich and Michael Levy for computational assistance. Support for this research has been provided by the US Department of Energy's SciDAC program under grants DE-FG02-07ER64446 and DE-SC0006684. We also thank DOE for support through the LANL/LDRD program.

Edited by: P. Jöckel

\section{References}

Colella, P. and Sekora, M. D.: A limiter for PPM that preserves accuracy at smooth extrema, J. Comput. Phys., 227, 7069-7076, 2008.

Colella, P. and Woodward, P. R.: The Piecewise Parabolic Method (PPM) for gas-dynamical simulations, J. Comput. Phys., 54, 174-201, 1984.

Davies, T., Cullen, M. J. P., Malcom, A. J., Mawson, M. H., Staniforth, A., White, A. A., and Wood, N.: A new dynamical core for the Met Office's global and regional modelling of the atmosphere, Quart. J. Roy. Meteor. Soc., 131, 1759-1782, 2005.

Dennis, J. M., Edwards, J., Evans, K. J., Guba, O., Lauritzen, P. H., Mirin, A. A., St-Cyr, A., Taylor, M. A., and Worley, P. H.: CAMSE: A scalable spectral element dynamical core for the Community Atmosphere Model, Int. J. High Perf. Comput. Appl., 26, 74-89, 2012.

Grinstein, F. F., Margolin, L. G., and Rider, W.: Implicit Large Eddy Simulation, Cambridge, 546 pp., 2007.

Jablonowski, C. and Williamson, D. L.: The pros and cons of diffusion, filters and fixers in atmospheric general circulation models, in: Numerical Techniques for Global Atmospheric Models, edited by: Lauritzen, P. H., Jablonowski, C., Taylor, M. A., and Nair, R. D., Springer, 381-493, 2011.

Jablonowski, C., Lauritzen, P. H., Nair, R. D., and Taylor, M.: Idealized test cases for the dynamical cores of atmospheric general circulation models: A proposal for the NCAR ASP 2008 summer colloquium, Tech. Rep., 74 pp., available at: http://www-personal.umich.edu/ $\sim$ cjablono/NCAR ASP_2008_idealized_testcases_29May08.pdf, 2008.

Kent, J., Jablonowski, C., Whitehead, J. P., and Rood, R. B.: Assessing tracer transport algorithms and the impact of vertical resolution in a finite-volume dynamical core, Mon. Weather Rev., 140, 1620-1638, 2012.

Lauritzen, P. H. and Thuburn, J.: Evaluating advection/transport schemes using interrelated tracers, scatter plots and numerical mixing diagnostics, Quart. J. Roy. Meteor. Soc., 138, 906-918, 2012.

Lauritzen, P. H., Skamarock, W. C., Prather, M. J., and Taylor, M. A.: A standard test case suite for two-dimensional linear transport on the sphere, Geosci. Model Dev., 5, 887-901, doi:10.5194/gmd-5-887-2012, 2012.

Lax, P. D. and Wendroff, B.: Systems of conservation laws, Commun. Pure Appl. Math., 13, 217-237, 1960.

Levy, M. N., Nair, R. D., and Tufo, H. M.: High-order Galerkin method for scalable global atmospheric models, Comput. Geosci., 33, 1022-1035, 2007.

Lin, S. J.: A "vertically Lagrangian" finite-volume dynamical core for global models, Mon. Weather Rev., 132, 2293-2307, 2004. 
Lin, S. J. and Rood, R. B.: Multidimensional flux-form semiLagrangian transport schemes, Mon. Weather Rev., 124, 20462070, 1996.

Lin, S. J. and Rood, R. B.: An explicit flux-form semi-Lagrangian shallow water model on the Sphere, Quart. J. Roy. Meteor. Soc., 123, 2477-2498, 1997.

Mahlman, J. D. and Sinclair, R. W.: Tests of various numerical algorithms applied to a simple trace constituent air transport problem, in: Fate of Pollutants in the Air and Water Environments, edited by: Suffet, I. H., Wiley, 8, 223-252, 1977.

Mason, P. J.: Large-eddy simulation: A critical review of the technique, Quart. J. Roy. Meteor. Soc., 120, 1-26, 1994.

Miura, H.: An upwind-biased conservative advection scheme for spherical hexagonal-pentagonal grids, Mon. Weather Rev., 135, 4038-4044, 2007.

Nair, R. D. and Jablonowski, C.: Moving vortices on the sphere: a test case for horizontal advection problems, Mon. Weather. Rev., 136, 699-711, 2008.

Nair, R. D. and Lauritzen, P. H.: A class of deformational flow test cases for linear transport problems on the sphere, J. Comput. Phys., 229, 8868-8887, 2010.

Nair, R. D. and Machenhauer, B.: The mass-conservative cellintegrated semi-Lagrangian advection scheme on the sphere, Mon. Weather. Rev., 130, 649-667, 2002.

Neale, R. B., Chen, C.-C., Gettelman, A., Lauritzen, P. H., Park, S., Williamson, D. L., Conley, A. J., Garcia, R., Kinnison, D., Lamarque, J.-F.,Marsh, D., Mills, M., Smith, A. K., Tilmes, S., Vitt, F., Cameron-Smith, P., Collins, W. D., Iacono, M. J., Rasch, P. J., and Taylor, M. A.: Description of the NCAR Community Atmosphere Model (CAM 5.0), NCAR Tech. Note NCAR/TN486+STR, 268 pp., 2010.

Niwa, Y., Tomita, H., Satoh, M., and Imasu, R.: A threedimensional icosahedral grid advection scheme preserving monotonicity and consistency with continuity for atmospheric tracer transport, J. Meteor. Soc. Japan, 89, 255-268, 2011.

Ovtchinnikov, M. and Easter, R. C.: Nonlinear advection algorithms applied to interrelated tracers: Errors and implications for modelling aerosol-cloud interactions, Mon. Weather Rev., 137, 632644, 2009.

Prather, M. J., Zhu, X., Strahan, S. E., Steenrod, S. D., and Rodriguez, J. M.: Quantifying errors in trace species transport modelling, PNAS, 150, 19617-19621, 2008.

Rancic, M., Purser, R. J., and Mesinger, F. A global shallow-water model using an expanded spherical cube: Gnomonic versus conformal coordinates, Quart. J. Roy. Meteor. Soc., 122, 959-982, 1996.

Roeckner, E., Bauml, G., Bonaventura, L., Brokopf, R., Esch, M., Hagemann, M. G. S., Kirchner, I., Kornblueh, L., Manzini, E., Rhodin, A., Schlese, U., Schulzweida, U., and Tompkins, A.: The atmospheric general circulation model ECHAM5. Part I model description, Tech. Rep. 349, Max Planck Institute for Meteorology, 127 pp., available at: http://www.mpimet.mpg.de/en/ wissenschaft/modelle/echam/echam5.html, 2003.

Rood, R. B.: Numerical advection algorithms and their role in atmospheric transport and chemistry models, Rev. Geophys., 25, 71-100, 1987.

Sadourny, R.: Conservative finite-difference approximations of the primitive equations on quasi-uniform spherical grids, Mon. Weather Rev., 100, 136-144, 1972.
Satoh, M., Matsuno, T., Tomita, H., Miura, H., Nasuno, T., and Iga, S.: Nonhydrostatic icosahedral atmospheric model (NICAM) for global cloud resolving simulations, J. Comput. Phys., 227, 34863514, 2008.

Staniforth, A. and Thuburn, J.: Horizontal grids for global weather and climate prediction models: a review, Quart. J. Roy. Meteor. Soc., 138, 1-26, 2012.

Taylor, M. A.: Conservation of Mass and Energy for the Moist Atmospheric Primitive Equations on Unstructured Grids in: Numerical Techniques for Global Atmospheric Models, edited by: Lauritzen, P. H., Jablonowski, C., Taylor, M. A. and Nair, R. D., Springer, 357-380, 2011.

Taylor, M. A., Tribbia, J., and Iskandarani, M.: The spectral element method for the shallow water equations on the sphere, J. Comput. Phys., 130, 92-108, 1997.

Taylor, M. A., Edwards, J., and St.-Cyr, A.: Petascale atmospheric models for the community climate system model: new developments and evaluation of scalable dynamical cores, J. Phys., 125, 1-10, 2008.

Taylor, M. A., St.-Cyr, A. and Fournier, A.: A non-oscillatory advection operator for the compatible spectral element method, Computational Science ICCS 2009 Part II, Lecture Notes In Computer Science, 5545, 273-282, 2009.

Thuburn, J.: Multidimensional flux-limited advection schemes, J. Comput. Phys., 23, 74-83, 1996.

Thuburn, J.: Some conservation issues for the dynamical cores of NWP and climate models, J. Comput. Phys., 227, 3715-3730, 2008.

Ullrich, P. A., Lauritzen, P. H., and Jablonowski, C.: Geometrically Exact Conservative Remapping (GECoRe): regular latitudelongitude and cubed-sphere grids, Mon. Weather. Rev., 137, 1721-1741, 2009.

van Leer, B.: Towards the ultimate conservative difference scheme, III, Upstream-centered finite-difference schemes for ideal compressible flow, J. Comput. Phys., 23, 263-275, 1977.

Williamson, D. L.: The evolution of dynamical cores for global atmospheric models, J. Meteor. Soc. Japan, 85B, 241-269, 2007.

Williamson, D. L. and Rasch, P. J.: Two-dimensional semiLagrangian transport with shape preserving interpolation, Mon. Weather Rev., 117, 102-129, 1989.

Williamson, D. L. and Rasch, P. J.: Water vapor transport in the NCAR CCM2, Tellus, 46A, 34-51, 1994.

Williamson, D. L., Drake, J. B., Hack, J. J., Jakob, R., and Swarztrauber, P. N.: A standard test set for numerical approximations to the shallow water equations in spherical geometry, J. Comput. Phys., 102, 211-224, 1992.

Zerroukat, M., Wood, N., and Staniforth, A.: (SLICE): a SemiLagrangian Inherently Conserving and Efficient scheme for transport problems, Quart. J. Roy. Meteor. Soc., 128, 2801-2820, 2002.

Zerroukat, M., Wood, N., and Staniforth, A.: A monotonic and positive-definite filter for a Semi-Lagrangian Inherently Conserving and Efficient (SLICE) scheme, Quart. J. Roy. Meteor. Soc., 131, 2933-2936, 2005. 